\title{
CORRECTION
}

\section{Correction to: First-year dispersal in white-tailed eagles Haliaeetus albicilla}

\author{
D. Rymešová ${ }^{1}$ R. Raab ${ }^{2} \cdot$ V. Machálková ${ }^{1}$ D. Horal ${ }^{3,4} \cdot$ D. Dorňáková ${ }^{1}$ - L. Rozsypalová ${ }^{1}$ P. Spakovszky ${ }^{2}$ I. Literák ${ }^{1}$
}

Published online: 16 July 2021

(c) Springer-Verlag GmbH Germany, part of Springer Nature 2021

Correction to: European Journal of Wildlife Research. https://doi.org/10.1007/s10344-021-01490-6

There were quite obvious typographical errors made in the Tables 4 and 6 during publishing process. Commas separating thousands in English were added very chaotically to numbers in the Tables 4 and 6 in the columns: MCP100, MCP95, KDE80, KDE50 and March MCP100. For example, there was stated 10,6720 instead of the right form 106,720 (alternatively 106720 or 106,720) in the first row and MCP100 column in the Table 4. Some numbers in mentioned columns had not the comma at all (especially in KDE80 and KDE50 columns). Values could be misunderstood by readers. We publish the corrected tables.

The original article can be found online at https://doi.org/10.1007/ s10344-021-01490-6.
D. Rymešová
Rymesova.D@seznam.cz
R. Raab
rainer.raab@tbraab.at
V. Machálková
machalkova.verca@gmail.com
D. Horal
david.horal@seznam.cz
D. Dorňáková
Dornakova.Denisa@seznam.cz
L. Rozsypalová
lenka.rozsypalova@gmail.com
P. Spakovszky
peter.spakovszky@tbraab.at 
Table 4 Dispersal characteristics of 35 young white-tailed eagles tracked during the first year of life (individuals with a lost signal or that died before March 31 are marked in italic). Max dist. maximal dispersal distance from the native nest $(\mathrm{km})$, Max dist. date date of the most distant location from the native nest, $M C P$ / KDE size of minimum convex polygon / kernel density estimate computed from given $\%$ of locations $\left(\mathrm{km}^{2}\right)$, No. of TSAs number of individual temporary settlement areas, Mean TSA area mean size computed from all TSAs $\left(\mathrm{km}^{2}\right)$, PFA area post-fledging area size $\left(\mathrm{km}^{2}\right)$, Azimuth 1 azimuth to North between the most distant location and the native nest (in degrees), Azimuth 2 azimuth to North between the centroid of KDE50 polygon and the native nest

\begin{tabular}{|c|c|c|c|c|c|c|c|c|c|c|c|}
\hline Ring & Max dist & Max dist. date & MCP100 & MCP95 & KDE80 & KDE50 & No. of TSAs & Mean TSA area & PFA area & Azimuth 1 & Azimuth 2 \\
\hline BS0041 & 346 & 06.07. & 106720 & 102980 & 47802 & 15825 & 7 & 170 & 254 & 94 & 8 \\
\hline BS0042 & 233 & 28.10 & 27226 & 2797 & 1137 & 357 & 2 & 507 & 318 & 120 & 84 \\
\hline LX493 & 179 & 29.03. & 25622 & 13376 & 2196 & 865 & 2 & 134 & 332 & 70 & 311 \\
\hline LX494 & 185 & 25.03 & 40159 & 12775 & 1537 & 565 & 1 & 132 & 269 & 315 & 312 \\
\hline LX495 & 160 & 30.01 & 20028 & 4503 & 911 & 295 & 1 & 1318 & 865 & 126 & 206 \\
\hline LX496 & 163 & 06.03. & 17177 & 14337 & 5142 & 1723 & 2 & 2277 & 1316 & 150 & 168 \\
\hline LX472 & 239 & 29.12 . & 20836 & 18842 & 6695 & 2630 & 2 & 276 & 26 & 111 & 103 \\
\hline LX473 & 347 & 25.02. & 85561 & 55003 & 25500 & 8747 & 4 & 300 & 18 & 270 & 299 \\
\hline LX475 & 123 & 18.08 & 15014 & 2980 & 963 & 318 & 0 & - & 1963 & 184 & 315 \\
\hline H0581 & 107 & 31.08 & 15962 & 2178 & 754 & 264 & 1 & 1737 & 1238 & 39 & 255 \\
\hline H0582 & 176 & 30.12 & 12359 & 10719 & 5437 & 1739 & 3 & 419 & 560 & 236 & 227 \\
\hline H0584 & 111 & 24.12 & 14645 & 14104 & 5877 & 2304 & 3 & 1029 & 1294 & 215 & 162 \\
\hline LX460 & 433 & 30.03 & 70533 & 24067 & 6961 & 2143 & 1 & 735 & 2383 & 301 & 264 \\
\hline LX462 & 204 & 13.10 & 59991 & 43856 & 6572 & 2148 & 3 & 663 & 61 & 226 & 258 \\
\hline H0585 & 93 & 31.03 & 2777 & 2205 & 447 & 138 & 2 & 471 & 149 & 183 & 332 \\
\hline LX305 & 110 & 21.01 & 9494 & 6247 & 2327 & 947 & 1 & 1263 & 383 & 120 & 120 \\
\hline LX465 & 126 & 28.02 & 12829 & 2213 & 812 & 253 & 1 & 947 & 5 & 236 & 189 \\
\hline LX463 & 373 & 21.03 & 37419 & 5733 & 3846 & 1288 & 1 & 846 & 2645 & 249 & 79 \\
\hline no ring & 324 & 23.11 & 63507 & 59177 & 23322 & 6378 & 3 & 330 & 128 & 173 & 182 \\
\hline H0624 & 193 & 24.03. & 24488 & 21240 & 7956 & 2963 & 3 & 521 & 0.4 & 170 & 182 \\
\hline LX628 & 293 & 14.08. & 44779 & 12524 & 2576 & 1001 & 3 & 442 & 426 & 305 & 182 \\
\hline LX629 & 187 & 30.03 & 39135 & 24635 & 3753 & 1137 & 2 & 2120 & 2963 & 298 & 186 \\
\hline LX621 & 366 & 31.03. & 61681 & 40489 & 4307 & 1594 & 1 & 2208 & 357 & 173 & 138 \\
\hline LX622 & 214 & 21.03 & 41495 & 19036 & 3393 & 1375 & 1 & 456 & 14 & 283 & 275 \\
\hline LX744 & 113 & 17.12 & 17457 & 16945 & 2094 & 625 & 4 & 330 & 150 & 173 & 155 \\
\hline LX742 & 159 & 15.02 & 35115 & 3969 & 852 & 284 & 1 & 418 & 627 & 267 & 286 \\
\hline LX852 & 123 & 11.03 & 10282 & 3077 & 1012 & 299 & 1 & 791 & 2289 & 3 & 269 \\
\hline LX853 & 216 & 12.09 & 26888 & 9474 & 1150 & 393 & 1 & 97 & 1537 & 299 & 216 \\
\hline H0349 & 339 & 24.12 & 111249 & 103497 & 32427 & 10648 & 5 & 1325 & 986 & 219 & 222 \\
\hline BSO044 & 236 & 31.08. & 17421 & 3065 & 1315 & 469 & 0 & - & 331 & 340 & 183 \\
\hline$L X 471$ & 249 & 30.08 & 28457 & 23297 & 13098 & 5408 & 1 & 108 & 415 & 282 & 286 \\
\hline LX193 & 183 & 26.09. & 16621 & 12279 & 3788 & 1216 & 2 & 676 & 1648 & 281 & 272 \\
\hline H0583 & 90 & 22.09. & 8284 & 4497 & 1190 & 410 & 0 & - & 2886 & 228 & 121 \\
\hline$L X 743$ & 413 & 05.09 & 34713 & 33310 & 15458 & 6554 & 1 & 2070 & 0.2 & 349 & 2 \\
\hline HO588 & 327 & 14.10 & 62852 & 60807 & 20875 & 7627 & 3 & 359 & 13 & 97 & 85 \\
\hline
\end{tabular}


Table 6 Final occurrence characteristics of tagged whitetailed eagles based on all March locations. (Individuals not surviving until March 31 are marked in italic, and most their values could not be computed). March centroid direction azimuth to North between the centroid of March MCP100 and the native nest, March centroid distance distance between the centroid of March MCP100 and the native nest, March MCP100 / total MCP100 individual proportion of March MCP100 out of total MCP100 computed from all obtained locations, March MCP100 size of the $100 \%$ minimum convex polygon computed from March locations, Last return $<200 \mathrm{~m}$ $1<5 \mathrm{~km}$ date of the last occurence closer than $200 \mathrm{~m} /$ $5 \mathrm{~km}$ from the native nest

\begin{tabular}{|c|c|c|c|c|c|c|}
\hline Ring & $\begin{array}{l}\text { March cen- } \\
\text { troid direction } \\
\left({ }^{\circ}\right)\end{array}$ & $\begin{array}{l}\text { March centroid } \\
\text { distance }(\mathrm{km})\end{array}$ & $\begin{array}{l}\text { March MCP } \\
\text { / total MCP100 } \\
(\%)\end{array}$ & $\begin{array}{l}\text { March } \\
\text { MCP100 } \\
\left(\mathrm{km}^{2}\right)\end{array}$ & $\begin{array}{l}\text { Last } \\
\text { return }<200 \mathrm{~m}\end{array}$ & $\begin{array}{l}\text { Last } \\
\text { return }<5 \mathrm{~km}\end{array}$ \\
\hline BS0041 & 142 & 72 & 18.7 & 19985 & 01.07. & 31.03. \\
\hline BS0042 & 304 & 36 & 16.0 & 4354 & 01.12 & 11.03. \\
\hline LX493 & 76 & 67 & 82.0 & 21016 & 06.07. & 24.03 . \\
\hline LX494 & 306 & 85 & 66.2 & 26593 & 05.07 & 05.03 . \\
\hline LX495 & 124 & 23 & 14.2 & 2854 & 05.03 . & 07.03 . \\
\hline LX496 & 155 & 61 & 22.1 & 3799 & 28.09. & 10.03. \\
\hline LX472 & 139 & 15 & 68.2 & 14209 & 21.07. & 22.09. \\
\hline LX473 & 292 & 179 & 2.4 & 2024 & 17.08. & 18.08. \\
\hline LX475 & 340 & 10 & 5.1 & 760 & 24.08 . & 31.03. \\
\hline H0581 & 358 & 19 & 47.0 & 7505 & 12.10 & 11.03. \\
\hline H0582 & 0 & 0 & 7.9 & 976 & 07.02. & 30.03 . \\
\hline H0584 & 108 & 29 & 16.1 & 2362 & 17.07. & 31.03. \\
\hline LX460 & 266 & 89 & 88.1 & 62128 & 01.10. & 02.10 . \\
\hline LX462 & 132 & 47 & 19.2 & 11548 & 05.09 & 05.09 . \\
\hline H0585 & 185 & 66 & 28.2 & 782 & 31.10 & 15.02. \\
\hline LX305 & 115 & 82 & 5.1 & 482 & 06.09. & 06.09. \\
\hline LX465 & 208 & 30 & 45.8 & 5872 & 14.08. & 14.08. \\
\hline LX463 & 241 & 179 & 83.4 & 31225 & 13.08 & 05.03 . \\
\hline no ring & 155 & 166 & 42.2 & 26831 & 27.09. & 28.09 \\
\hline H0624 & 180 & 116 & 30.3 & 7424 & 11.09. & 11.09. \\
\hline LX628 & 173 & 63 & 9.1 & 4074 & 16.08 & 10.11. \\
\hline LX629 & 301 & 100 & 49.7 & 19464 & 15.08 & 05.02 . \\
\hline LX621 & 167 & 178 & 35.6 & 21964 & 27.08. & 08.03 \\
\hline LX622 & 280 & 157 & 6.6 & 2745 & 05.09 & 15.09. \\
\hline LX744 & 323 & 39 & 23.9 & 4169 & 02.09 & 24.03. \\
\hline LX742 & 104 & 49 & 59.3 & 20818 & 10.09 & 28.03 \\
\hline LX852 & 349 & 39 & 64.4 & 6619 & 18.09. & 13.03. \\
\hline LX853 & 284 & 59 & 60.3 & 16218 & 10.09 & 23.03 . \\
\hline H0349 & 127 & 109 & 30.1 & 33513 & 23.08 & 31.03. \\
\hline BSO044 & - & - & - & - & 10.07 & 13.09. \\
\hline$L X 471$ & - & - & - & - & 07.08 & 22.08 \\
\hline LX193 & - & - & - & - & 10.08 & 12.12 \\
\hline$H 0583$ & - & - & - & - & 17.07. & 10.10 \\
\hline$L X 743$ & - & - & - & - & 31.08. & 31.08. \\
\hline H0588 & - & - & - & - & 24.06 & 05.08 \\
\hline
\end{tabular}

The original article has been corrected.

Publisher's Note Springer Nature remains neutral with regard to jurisdictional claims in published maps and institutional affiliations. 